\title{
Research on Application to College Study in Organic Chemistry Teaching based on Computer Software
}

Jing Wang

Teacher's College, Beihua University, Jilin City, China

\begin{abstract}
In order to give more accurate response to some basic chemical problems, it relates to the theoretical analysis and calculation, so the computer chemistry has been developed rapidly. The computational chemistry software has its unique properties, and it can achieve human-computer interaction, showing vividness and intuitiveness, etc. the effect is significant, which concrete teaching case analysis, and in chemistry teaching by making good use of computer chemistry software so as to improve the actual effect of classroom teaching, enrich the teaching contents in class, animate the classroom atmosphere and enhance students enthusiasm of learning chemistry.
\end{abstract}

\section{General instruction}

Development of computer technology in many fields and opportunities in the field of chemical, more specialized computer software into use these the use of software, by the chemical symbol or chemical formula for the expression of the image, etc., these symbols belong to the plane of the traditional symbols, but it is these symbols and chemical structures of all chemical information can express object of study can also be described in the microscopic world of chemical, chemical microscopic world is more esoteric, using computer software can well reveal because these two advantages, computer software is widely used not only in the scientific community, but also become one of the popular university teaching tools, and even become an essential teaching tool. Traditional teaching methods are mainly chemical Chemistry, models and slides, the disadvantage of this kind of teaching methods that can not be vividly describes the chemical issues to be told, and computer software chemistry teaching methods are able to solve this problem, the chemical when combining software with multimedia, vivid display the content to be described, some of the more abstract issues concrete, simplified, thereby reducing the difficulty of teaching students to understand abstract chemistry problem, no longer as difficult as using traditional methods, and can range from simple concrete steps begin to understand and improve their interest in chemistry learning, encourage them to continue in-depth understanding of chemistry microscopic world.

So far, the popular software on the market a variety of chemical, computational chemistry software, which is more commonly used are three categories, namely Caucasian developed Caucasian, Hypercube developed and developed by Cambridge Soft. Writer mainly from the actual teaching case of view, the computer software used in university chemistry organic chemistry teaching briefly described, and discuss issues related to the chemical for software design.

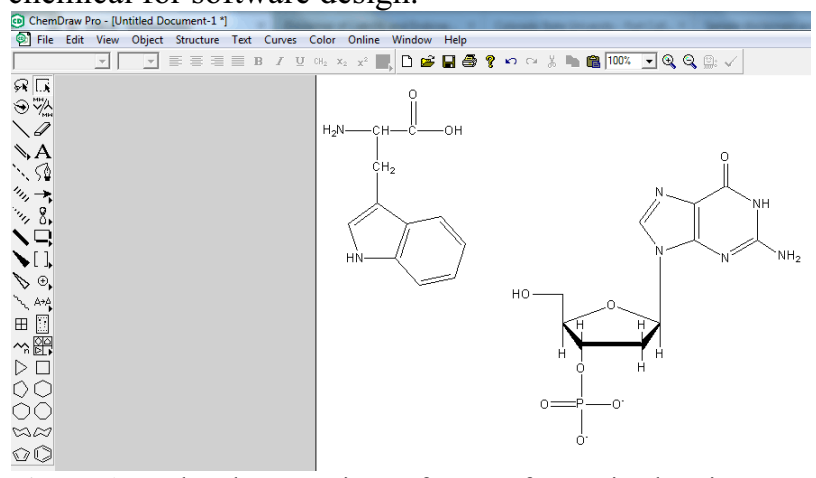

Figure 1. Molecular mapping software of organic chemistry

\section{The traditional higher mathematics education}

In traditional organic chemistry teaching, about threedimensional structure of the molecule to explain, just by Molecular Structure to show, but due to insufficient visual image, three-dimensional structure of the molecule for students is still obscure, difficult for students to imagine its structure and thus can not understand, but the structure of molecules of organic compounds is the focus of teaching, its three-dimensional geometry of the reaction mechanism and constitute the physical and chemical nature of the relationship to the molecule, etc. with the evolution of modern technology, there are many software can molecule three-dimensional structure of the simulation demonstration, teachers can use it for 
secondary education, so that students can clearly recognize intuitively structural model molecules, such as ChemOffice, Hyperchem, Gaussian 03 and so on chemically assisted instruction software, can be achieved by molecular stereoscopic 3D technology simulation demonstration structure for various models can simulate the structure of the molecule, such as stick models, metal bond model and sticks models, etc. Such software can not only enhance students 'understanding of molecular structure, but also improve students' spatial thinking ability and imagination.

For example, when teaching against ethane molecule conformation can be Gaussian 03 software to assist teaching, through its operation, it is conceivable molecular conformation, and can be associated with the energy of its molecules to their specific operation is as follows: the first step in the establishment of an ethane molecule model and optimize its molecular structure. Second step, in order to rotate 360 degrees CC, is necessary for the H4-C3-C2-H1 dihedral angle change for the third step, input scan to scan for potential energy curves of the rotation process. fourth step variation from $\mathrm{CC}$ rotation curve analysis of molecular potential energy conformation, and the lowest point potential energy curves corresponding conformation is stable conformations, also known as cross-type conformation; while the highest point of the potential energy curves corresponding conformation is overlapping conformation when teachers during teaching, but also to assist by designing courseware, these conformations shots, made a dynamic picture and set these figures too and potential energy curve movements simultaneously, so that students will be able to grasp very clearly understand the various conformations of ethane its corresponding potential relationship through these animations.

Analysis of the characteristics of organic matter

For some properties of organic compounds, such as physical and chemical properties, related chemical auxiliary software can simulate and predict, to help students achieve the image of intuitive understanding. For example, when studying the molecular orbital theory, can assist the relevant chemical teaching software to overcome difficult problems in the past, with the help of chemical auxiliary software to help students understand abstract theory of knowledge, so that students clearly observed by the shape of the molecule reaches orbit HOMO understand and grasp the concepts so abstract image of the theoretical knowledge into knowledge the approach not only reduces the burden on teachers teaching, but also reduces the difficulty students understand, you can clearly see the three-dimensional shape of the molecular orbital grasp. Hyperchem software also can be achieved by drawing on the molecular orbital, including its level presented the sketch; the specific steps are as follows:

First, the establishment of a molecular model is proper optimization. Secondly, by Compute following Orbital option to automatically generate the molecular orbital and the energy level schematic. Third in the schematic generated randomly select a track, come on its threedimensional models show this intuitive, obvious effect of three-dimensional hierarchical diagram fully demonstrated the conjugated $\pi$ bond-dimensional structure helps students improve learning efficiency.

The basic parameters for the structure of organic compounds and analysis of relevant physical and chemical properties, can also be related to chemical auxiliary software to achieve, for example, learning characteristics for acetyl salicylic acid, you can use the Chem Office software to assist its specific operation steps are as follows: first, first by Chem Draw molecular Structure to describe a second step, by clicking on the View menu option to let it calculate the corresponding compounds of critical pressure, critical temperature, critical volume, boiling point, melting point. Molecular weight generates heat and so on.

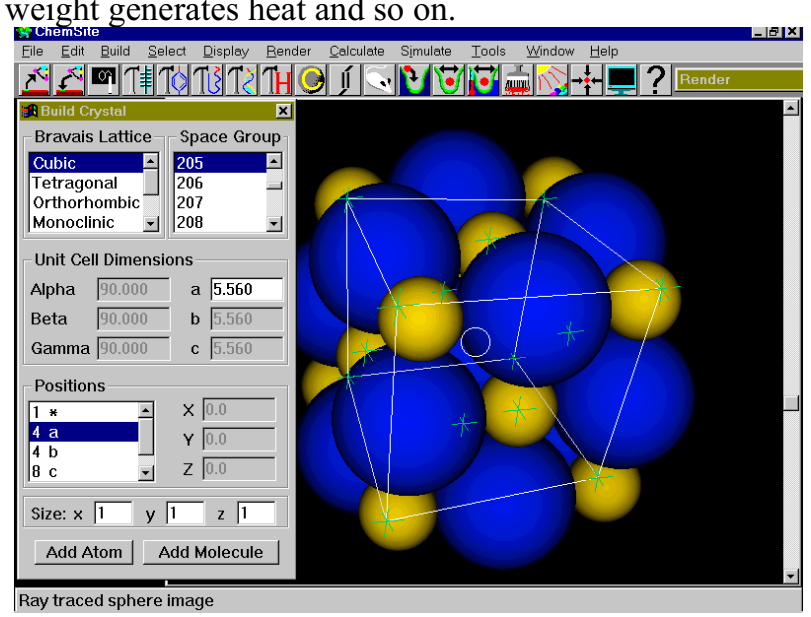

Figure 2. Molecular structure building software of organic chemistry

\section{The keyword computational design}

In organic chemistry teaching, the compound is also the focus of organic reactions, the mechanism and impact is very complex species are also very large, very abstract and difficult, often difficult for students to grasp the depth of their understanding, but for it to learn help students grasp the future synthesis process and the choice of a reasonable method to improve students' scientific research ability, so learning organic reactions is of great significance for students. Gaussian 03 software appears a good solution to this problem, so that students can easily understand and master this theory, for example, for learning bimolecular nucleophilic reactions, the specific steps are: a first step, to establish the appropriate $\mathrm{CH} 3 \mathrm{CL}$ $+\mathrm{BR}-$ and $\mathrm{CH} 3 \mathrm{CL}+\mathrm{Br}-$ molecular model, and its optimization the second step, by using the form QST2 related to the reaction, and the keyword corresponding computational design third step, to render the IRC by calculating the energy variation of the molecular structure and in the course of the reaction. After their reactions via software demo can be observed, the nucleophilic reagent, leaving the evolution of the group, between the central carbon atom, when the nucleophile bypass leaving group and slowly approaching the central carbon atom, it will forming $\mathrm{Br}-\mathrm{C}$, when the leaving group is Chemistry her away from the central carbon atom, then the $\mathrm{C}-\mathrm{Cl}$ presentation will slowly break phenomenon causes the central carbon atom and the corresponding changes occur, according to which hydrogen atoms leaving groups 
appear direction deflection when the reaction carried out as described above to the transition state, then the central carbon atom structure will change, mostly showing sp2 hybrid phenomenon, and it will work with a leaving group and the pro-nucleophile is on the same line, the other atom or a group will also appear in a plane perpendicular to this line until at last completely away leaving group other atoms, as an independent product and independent of, the nucleophilic reagent will combine to form a bond with the central carbon atom of these processes are through GaussView software image presentation and observed.

After their reactions via software demo can be observed, the nucleophilic reagent, leaving the evolution of the group, between the central carbon atom, when the nucleophile bypass leaving group and slowly approaching the central carbon atom, it will forming $\mathrm{Br}-\mathrm{C}$, when the leaving group is fChemistry her away from the central carbon atom, then the $\mathrm{C}-\mathrm{Cl}$ presentation will slowly break phenomenon causes the central carbon atom and the corresponding changes occur, according to which hydrogen atoms leaving groups appear direction deflection when the reaction carried out as described above to the transition state, then the central carbon atom structure will change, mostly showing sp2 hybrid phenomenon, and it will work with a leaving group and the pro-nucleophile is on the same line, the other atom or a group will also appear in a plane perpendicular to this line until at last completely away leaving group other atoms, as an independent product and independent of, the nucleophilic reagent will combine to form a bond with the central carbon atom of these processes are through GaussView software image presentation and observed, the use of this chemical auxiliary software for student learning plays a great role in improving student can therefore more in-depth understanding to grasp the concept of the transition state as well as organic reaction principle.

\section{The understanding of molecular spectroscopy analysis}

Learn about the molecular spectral characteristics of organic compounds can encourage students to be more flexible in order to analyze the perspective of molecular material world in modern society, the scientists want to achieve for the understanding of molecular spectroscopy analysis, usually by modern instruments molecule organics accurate analysis of the structure to achieve, but it is difficult for this type of instrument referenced test in real classrooms. while Gaussian 03 software can be easily implemented in the classroom to simulate and predict the molecular spectra, such as infrared spectroscopy for organic molecules and vibration when the mode of learning can refer to the software, the specific steps are as follows: a first step, to establish and optimize the corresponding molecular model the second step, to calculate the molecular vibration frequency table by Freq keyword. The third step is to identify the corresponding calculation result output file and open the fourth step, click Vibrations option to choose a pop of molecular vibration frequency, can be obtained through the StChemistry vibration modes corresponding amount of audio, and the molecular compound infrared spectra can be obtained by clicking on the spectrum. Such intuitive display vibration compounds through animation, to obtain the corresponding infrared spectra, etc. for students, it is very easy to learn to master.

In addition, the software can also ChemOffice mass to charge ratio of organic compounds and NMR spectra prediction and simulation, for example, for the simulation and prediction of the benzophenone, the specific steps are as follows: a first step, the establishment of appropriate in ChemDraw the molecular structure and optimize a second step, by clicking Predict 1H NMR Shifts derived $1 \mathrm{H}$ NMR chemical shift spectra and the corresponding form. Third step, by clicking Show Analysis Windows to get the corresponding compound of the mass to charge than the students by observing the software forecast shows more easily grasp the content to improve learning efficiency.

\section{Conclusions}

The overall goal of chemistry education, new curriculum standards referred to "the learning process of students in chemistry , rich visual, tactile and aesthetic experience, access to chemistry of enduring interest in learning, form the basic chemistry literacy", "improve the aesthetic ability to understand chemistry the unique role of cultural life and social development." Here is more clearly explained the ultimate goal of chemistry courses. In the "targets" in the four fields of study made specific teaching requirements, these instructional purposes basically not much difference with older versions of curriculum standards. It is worth mentioning that in the "comprehensive exploration of the area," the teaching objectives, calling for "understanding the relationship between chemistry and nature, chemistry and life, chemistry and culture, between chemistry and technology, to explore and comprehensive chemistry activities, and to published in various forms of learning outcomes ", where clearly pointed out a comprehensive exploration of objects and methods, while the new curriculum standards also require our chemistry curriculum to make students feel a sense of pleasure and success.

As we all know, in today's education, discipline increasingly fine division. Such an increase implies something the students want to learn more and more, but their time is fixed. As a result, the constant increase in core subjects or other subjects only make students more overwhelmed. For this reason, the development trend of the moment the best education is infinitely split from discipline to a new integration. Chemistry education in this, should implement the existing curriculum standard, advocated chemistry ability is to train students and humanities integration development. It is an integrated aesthetic intuition all sense and sensibility to chemistry ability into aesthetic activity. Therefore, how people perceive the aesthetic intuition, how to understand the 
process of aesthetic intuition in chemistry education's role, and what kind of approach to training is worth exploring issues such as students' aesthetic intuition.

See some natural or man-made works of chemistry, we tend to happen on an emotional excitement, perhaps pleasant excitement, perhaps sad passionate, whether it is the former or the latter, in short, we were moved, so emotional the excitement, called appreciation, that is, we are looking at things from the beauty. Education is when a person learned in school has forgotten the rest of the stuff. Into their own behaviours within that chemistry of the way of thinking is not visible knowledge is permanent. No matter what kind of chemistry education means education methods, we want to achieve the ultimate goal is to train a person's right or even aesthetic intuition is a national beauty bright positive psychological intuition and aesthetic.

Because aesthetic intuition without hesitation and a look that is beautiful aesthetic phenomenon, people tend to go for the emotional behaviours aesthetic intuition, put it on the shelf, and scientific and rational opposition. As Freud and psychoanalysis, led by western modernist chemistry is the chemistry expression of this anti-rational. After careful thought is not difficult to find, aesthetic intuition itself is permeated with a rational component, bears deep and rich social and cultural connotations, this intuition has all the ability to think. It has a rational component, needs to be guided chemistry education and promotion. Aesthetic intuition is an important measure of the strength of the aesthetic capacity through chemistry training of chemistry generally has relatively strong aesthetic intuition.

\section{References}

1. Chee Soon Juan, Zhang Yong, Zhao Yang, a chemical commonly used software, such as research and development of multimedia teaching software. Computers and Applied Chemistry, 45(2010)

2. Liu Xiaodong, Zong ball .GaussView some applications in chemistry teaching. University Chemistry, 37(2009)

3. Zheng Wenrui computational chemistry applications in chemistry and chemical engineering. Shanghai Chemical12(2010)

4. Chee Soon Juan, Zhang Yong, Zhao Yang, a chemical commonly used software, such as research and development of multimedia teaching software. Computers and Applied Chemistry, 34(2009)

5. Xiao Ying. Aesthetic strategies of Contemporary Aesthetic Culture. Academic Monthly58(2002)

6. Chen Meimin. Aesthetic under Contemporary Aesthetic Culture. Hainan Radio and Television University, 89(2005)

7. Lu Chang. On the relationship between Chemistry s education and aesthetic education. Theory and practice of education, 75(2002)

8. Chen Chi-yu. Chinese modern aesthetics and Chemistry theory . Central China Normal University, 124(2000) 